\title{
Levenberg-Marquart logistic deep neural learning based energy efficient and load balanced routing in MANET
}

\author{
A. Sangeetha ${ }^{1}$, T. Rajendran ${ }^{2}$ \\ ${ }^{1}$ Research Scholar, Periyar University, Salem, India \\ ${ }^{2}$ Kangeyam Arts and Science College, Bharathiar University, India
}

\begin{abstract}
Article Info
Article history:

Received Jan 2, 2021

Revised May 18, 2021

Accepted Jun 1, 2021

Keywords:

Energy utilization

Logistic activation function

Residual load and energy

Throughput

ABSTRACT

As the advent of new technologies grows, the deployment of mobile ad hoc networks (MANET) becomes increasingly popular in many application areas. In addition, all the nodes in MANET are battery operated and the node mobility affects the path stability and creates excessive traffic leads to higher utilization of energy, data loss which degrades the performance of routing. So, in this paper we propose Levenberg-Marquardt logistic deep neural learning based energy efficient and load balanced routing (LLDNL-EELBR) which is a machine learning method to deeply analyze the mobile nodes to calculate residual load and energy and it also uses logistic activation function to select the mobile node having higher residual energy and residual load to route the data packet. Experimental evaluations of three methods (LLDNLEELBR, multipath battery and mobility-aware routing scheme (MBMAOLSR) and opportunistic routing with gradient forwarding for MANETs (ORGMA)) were done and the result reveals that LLDNL-EELBR method is able to increase the throughput and minimizes the delay and energy consumption in MANET when compared to works under consideration.
\end{abstract}

This is an open access article under the CC BY-SA license.

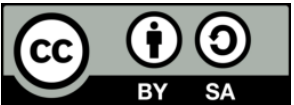

Corresponding Author:

A. Sangeetha

Department of Computer science

Government Arts and Science College

Modakkurichi, Erode, India

Email: sangeethakng@gmail.com

\section{INTRODUCTION}

The digital revolution in the last decade has opened a gate way of new opportunities where MANET plays a vital role in-case of disaster to traditional communication models i.e., mobile networks and internet crisis. MANET is an unstructured network of mobile nodes connected via radio links. Every device in MANET moves in a random direction and changes their connections to other device to route data packets. The unbalanced load distribution among nodes directed to a power dissipation which causes link failure and affects the network performance. Therefore, energy and load balanced routing is an important problem. Several researches have been done to perform energy efficient and load balanced routing. But it failed to decrease the time and energy consumption during data packet routing and data delivery. When transmitting data from source to destination, the residual energy and stability of the link must be analyzed. The residual energy is calculated by subtracting total transmission energy (sum of receiving energy and transmitting energy) from initial energy of the node. During data transmission, the route discovery process finds out the node with higher residual energy. If the nodes residual load is low, the data transmission process becomes very slow, and it automatically causes traffic which decreases the throughput. Hence, to increase throughput and extend the network lifetime, both residual energy and load is very essential. 
A multipath battery and mobility-aware routing scheme [1] was designed to choose the stable paths to destination. But this scheme failed to reduce the end-to-end delay. A reliable and practical opportunistic routing protocol for MANETs [2] increases packet delivery ratio whereas energy consumption during data packet routing was not reduced. A forwarding strategy was introduced in [3] to reduce the topology control traffic. However, the routing time was not reduced. Bandwidth-satisfied and coding-aware routing protocol [4] reduces the total bandwidth consumption; the routing efficiency was not improved. Secure optimized link state routing protocol [5] finds a secure link for the routing. However, this system does not consider any external attacks during packet transmission. MEQSA-OLSRv2 [6] solves the problem of limited energy resources, node mobility and network traffic in MANET and wireless sensor network (WSN). However, throughput was lower. A particle swarm optimization approach [7] carries out energy-optimized routing in MANET. But packet delivery ratio was not improved. In least common multiple based routing [8] for load-balancing in MANET, packet loss rate was not solved. A power and load-aware routing scheme based on the dynamic source routing (DSR) protocol scheme [9] increases the operational life of nodes and the network lifetime. But energy utilization was higher. A novel approach was introduced to increase the network performance through distribution of load among the free nodes, but stable, reliable and load balanced routing was not performed [10]-[12]. A stable and energy efficient routing algorithm [13] with application of learning automata theory results in lower packet delivery ratio.

A novel technique was introduced in [14], [15] with the intention of increasing load balancing capacity. But the number of data lost was higher and network lifetime was not improved. Joint cost and secured node disjoint energy-efficient multipath routing [16] improves security in data routing and reduces energy utilization and execution time during multipath routing. However, performance of energy efficiency is not sufficient. Minimal energy consumption with optimized routing [17] uses minimal energy during packet transmission. However, it increases the execution time. An intelligent energy-aware routing protocol for MANET [18] did not consider the load of nodes during transmission. Reliable and energy efficient protocol depending on distance and remaining energy [19] reduces energy consumption. But it failed in terms of packet delivery ratio. An Ad Hoc on demand multipath distance vector routing protocol using fitness function [20] finds out the optimal path from source to destination. But it failed to address energy consumption and network lifetime. Afsar and Younisin [21], Maurya et al. [22], cross-layer design for WSNs with energy scavenging and transfer capabilities and delay aware energy efficient routing were introduced to reduce the energy utilization. However, energy efficiency of these methods was not sufficient. Secure energy efficient routing protocol [23] selects a secure route path. But it failed to attain minimal packet loss rate. A clustering approach and energy efficient technique was implemented in [24], [25] for increasing the network lifetime by considering the mobility, energy of mobile nodes and redundant packet generation. However, the energy efficiency is not sufficient. To overcome the above-mentioned issues such as higher energy consumption during data transmission, lesser packet delivery ratio and network lifetime, LLDNL-EELBR method is introduced in this paper. The structure of the paper is planned is being as: section 2 depicts research methods. Section 3 explores result and discussion. In section 4, the conclusion of this paper is presented.

\section{RESEARCH METHOD}

\subsection{Problem formulation}

In MANET, each and every node act as a host and router. Due to error-prone wireless connections, dynamic changing structure, data routing without any disconnections is a complex task. Furthermore, an intermediate node is utilized for longer duration results in traffic congestion. This traffic causes higher latency and reduces the energy of nodes. Thus, energy efficient and load balanced data routing is an important issue to be considered. Therefore, LLDNL-EELBR method is introduced by using LevenbergMarquardt logistic deep neural learning concepts which prolongs network lifetime and increases throughput in MANET by selecting nodes with higher residual energy and residual load for routing.

\subsection{The proposed LLDNL-EELBR method}

In LLDNL-EELBR method, LLDFFNL algorithm attains greater consideration because it is fast and requires relatively less computational time and is very useful for real-world applications. The LLDFFNL is an artificial intelligence function that employs the workings of human brain to find out the best mobile nodes. This method comprises of four layers, namely one input layer, two hidden layer and one output layer.

Figure 1 shows the structure of LLDFFNL algorithm in which MANET is organized in the form of graphical representation as ' $g(A, B)$ '. Here, ' $A$ ' represents a total number of mobile nodes ' $\tau_{i}=\tau_{1}, \tau_{2}, \tau_{3}, \ldots, \tau_{n}$ ' dispensed in a square area of ' $X * Y$ ' within the communication range ' $r$ ' and ' $B$ ' indicates a connection between nodes in the network. Among the several mobile nodes, LLDNL-EELBR method identifies energy efficient 
and load balanced mobile nodes and route the data packets. LLDFFNL consists of several layers and each layer in LLDFFNL utilizes the output from one layer as input to the next layer and deep indicates the number of layers. Further, LLDFFNL contains a substantial credit assignment path (CAP) depth. CAP explains causal connection between the input and output layer. Depth of the CAP is the number of hidden layers plus one. LLDFFNL is a feedforward network in which data flow from input layer to output layer without backpropagation. It constructs a map of virtual neurons with mobile nodes and initializes random weights to each node. The input layer receives the number of mobile nodes as input. After obtaining input, hidden layers deeply examines each node and calculates their residual energy and load which is sent to the output layer. The weights and these inputs are multiplied in the output layer and give a prediction result ' 0 ' or ' 1 ' using a logistic activation function. If the network didn't exactly find outs the best nodes, an LLDFFNL adjusts the weights based on their error. We use the root mean squared method to reduce errors. Moreover, the backpropagation is applied in LLDFFNL to train the multilayer neural network by changing the weights in the nodes to improve routing performance according to the error correction learning function. In this manner, the LLDFFNL accurately selects the best nodes in MANET to obtain reliable data routing.

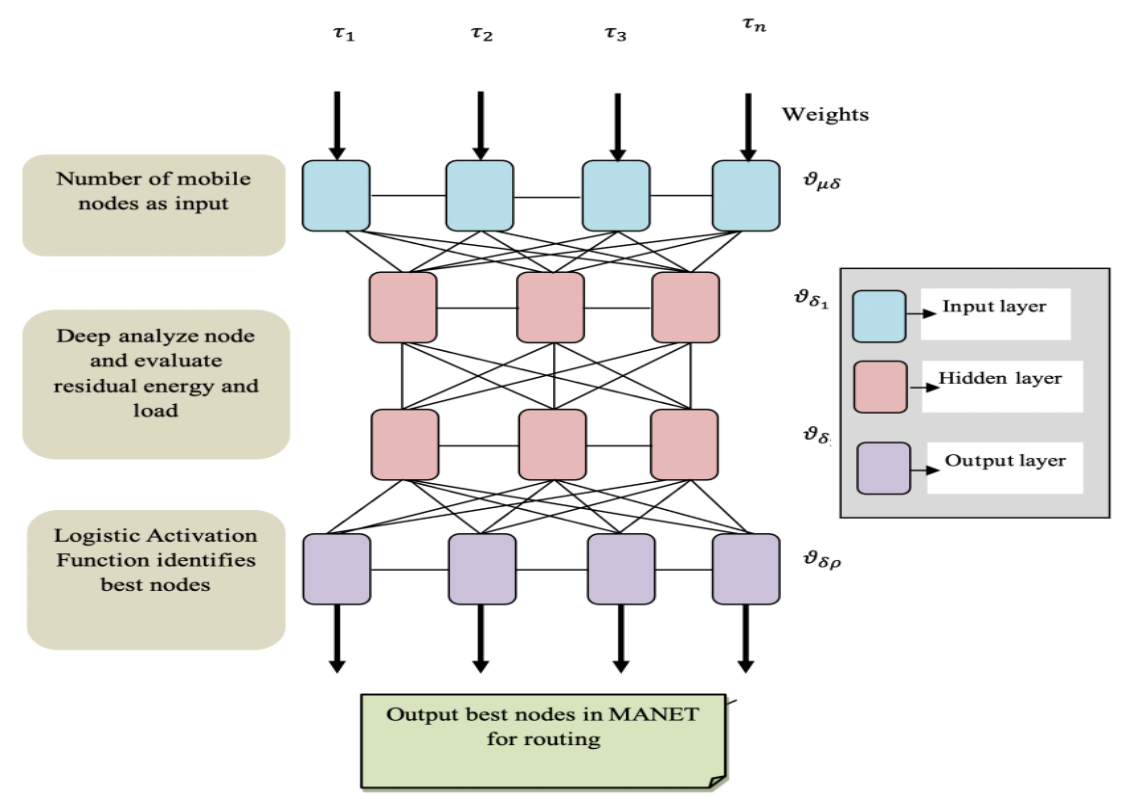

Figure 1. Structure of LLDFFNL algorithm

Figure 2 depicts a typical MANET network with 8 nodes. The data has to be transmitted from node a (source) to node $\mathrm{H}$ (destination) through an optimized path. The first step identifies the eligible nodes through LLDN-EELBR methodology. Among the eligible nodes, an efficient path would be identified and data will be transferred through this path.

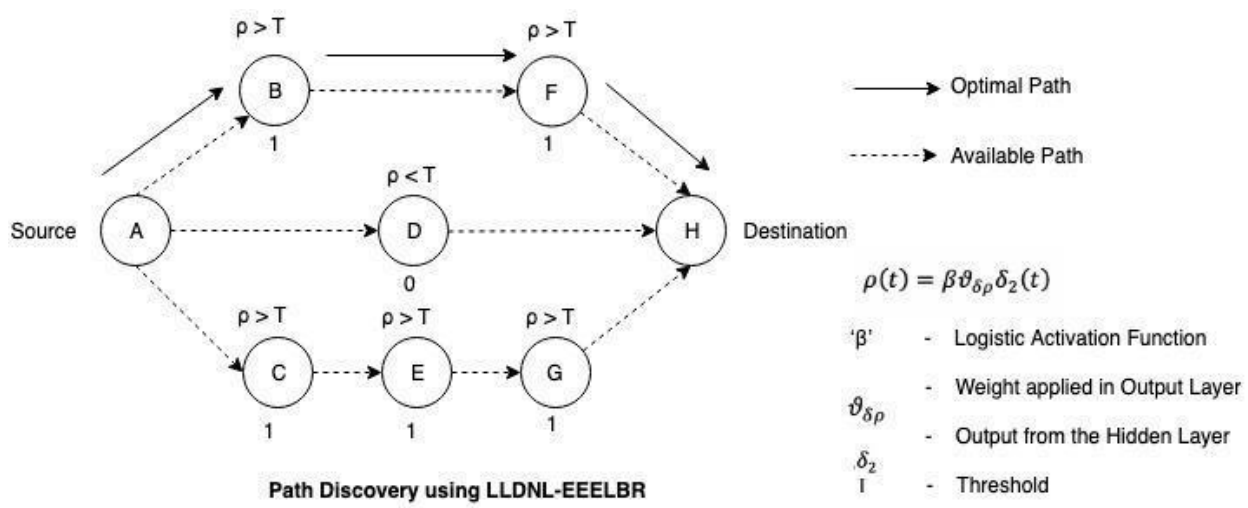

Figure 2. Path discovery in LLDFFNL algorithm 
Let us consider the mobile nodes ' $\tau_{i}=\tau_{1}, \tau_{2}, \tau_{3}, \ldots, \tau_{n}$ ' as input. Thus, the neurons operation in the input layer ' $(\mu)$ ' obtained as,

$$
\mu(t)=\sum \tau_{i} \vartheta_{\mu \delta}+\gamma_{j}
$$

From $(1)$, ' $\mu(t)$ ' represents neuron activities in input layer at a time ' $t$ ' and ' $\tau_{i}$ ' denotes an input mobile node and ' $\vartheta_{\mu \rho}$ ' refers to weight between the input and hidden layer and ' $\gamma_{j}$ ' indicates the bias term. After getting the input, the neuron in input layer ' $\mu$ ' combine an input mobile node ' $\tau_{i}$ ' with weights ' $\vartheta_{\mu \rho}$ ' and bias term ' $\gamma_{j}$ '. Consequently, the input layer forward input mobile nodes to the hidden layer. In LLDFFNL, the first hidden layer determines the residual energy of each input mobile node using expression,

$$
\delta_{1}(t)=\sum \mu(t) \vartheta_{\delta_{1}}, \alpha_{\tau_{i}}
$$

From (2), ' $\delta_{1}(t)$ ' refers to output of first hidden layer at time ' $t$ '. Here, ' $\mu(t)$ ' represents the input acquired from the input layer i.e. number of mobile nodes ' $\tau_{i}=\tau_{1}, \tau_{2}, \tau_{3}, \ldots, \tau_{n}$ ' whereas ' $\vartheta_{\delta_{1}}$ ' is the weight of first hidden layer and ' $\alpha_{\tau_{i}}$ ' denotes the residual energy of mobile nodes. Whenever the residual energy of mobile nodes is minimal, link failure occurs and therefore the data packet routing is failed. From that, identification of higher residual energy is essential to attain the reliable data transmission. The residual energy of mobile node ' $\alpha_{\tau_{i}}$ ' is calculated as,

$$
\alpha_{\tau_{i}}=\alpha_{I}-\left(\alpha_{T}+\alpha_{R}\right)
$$

From (3), ' $\alpha_{I}$ ' is the initial energy of mobile node ' $\tau_{i}$ ' and ' $\alpha_{T}$ ', ' $\alpha_{R}$ ' signifies energy utilized by a node to transmit and receive the data packets. Thus, the amount of energy consumed by a node ' $\alpha_{U}$ ' to send and receive packets is mathematically measured using as,

$$
\alpha_{U}=T \alpha_{\tau_{i}}-\alpha_{\tau_{i}}
$$

From (4), ' $T \alpha_{\tau_{i}}$ ' represent the total energy level of a mobile node. Next, the residual energy of all mobile nodes is sent to the second hidden layer. The residual load of mobile node is computed in the second hidden layer ' $\delta_{2}(t)$ ' at time ' $t$ ' using,

$$
\delta_{2}(t)=\sum \delta_{1}(t) \vartheta_{\delta_{2}}, R \varphi_{\tau_{i}}
$$

From (5), ' $\delta_{2}(t)$ ' indicates the output of second hidden layer and ' $\delta_{1}(t)$ ' is the residual energy which is obtained from the first hidden layer. Here ' $\vartheta_{\delta_{2}}$ ' represents the weight of second hidden layer and ' $R \varphi_{\tau_{i}}$ ' refers to residual load of nodes. The load on each mobile node ' $\varphi_{\tau_{i}}$ ' is calculated as,

$$
\varphi_{\tau_{i}}=\frac{n_{\tau_{i}}}{T_{\tau_{i}}}
$$

From (6), ' $T_{\tau_{i}}$ ' represent the total load capacity of mobile node and ' $n_{\tau_{i}}$ ' indicates that the amount of data packets being carried by node ' $\tau_{i}$ '. Followed by, the residual load of a node is estimated using as,

$$
R \varphi_{\tau_{i}}=T_{\tau_{i}}-n_{\tau_{i}}
$$

From (7), the residual load is calculated for each mobile node ' $\tau_{i}$ ' in network. Subsequently, the hidden layer sends residual energy and residual load results to the output layer. The best mobile nodes are selected in output layer using expression,

$$
\rho_{i}(t)=\beta_{i} \vartheta_{\delta \rho} \delta_{2}(t)
$$

From (8), ' $\rho_{i}(t)$ ' represents output layer result at time ' $t$ ' and ' $\vartheta_{\delta \rho}$ ' indicates the weight between the hidden and output layer and ' $\beta_{i}$ ' is the logistic activation function. The LLDNL-EELBR Technique used the logistic regression function as activation function algorithms to increase the routing efficiency in MANET. The logistic activation function ' $\beta_{i}$ ' result is mathematically formulated as, 


$$
\beta_{i}=\left\{\begin{array}{c}
\text { if }\left(\alpha_{\tau_{i}} \text { and } R \varphi_{\tau_{i}}>T\right), \text { then } \rho_{i}(t)=1 \\
\text { otherwise }, \rho_{i}(t)=0
\end{array}\right.
$$

From (9), ' $T$ 'represent threshold value assigned for residual energy and residual load. In LLDFFNL, the output of logistic sigmoid function ranges between ' 0 ' and ' 1 '. The output of the logistic activation function is depicted in Figure 3.

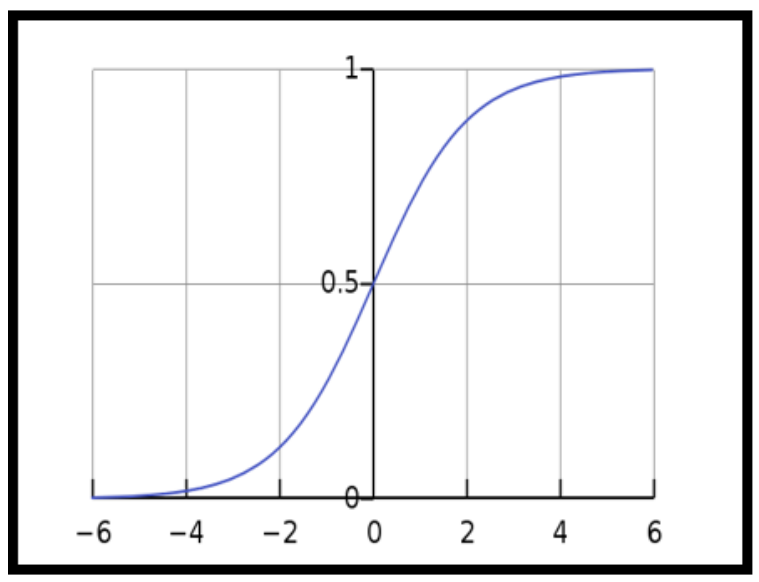

Figure 3. Logistic activation function output

From that, the output layer result is mathematically obtained as,

$$
\rho_{i}(t)=\left\{1, \tau_{i} \text { isselected for routing } 0, \tau_{i}\right. \text { is not selected for routing }
$$

From (10), ' $\rho_{i}(t)=1$ ' indicates that the mobile node ' $\tau_{i}$ 'selected to perform data routing whereas ' $\rho_{i}(t)=0$ ' signifies the mobile node ' $\tau_{i}$ ' is not chosen. For each prediction result of mobile node, the error is calculated as differentiation between target output ' $\rho_{i}$ ' $(t)$ ' and obtained output ' $\rho_{i}(t)$ ' using root-meansquared error using,

$$
\operatorname{RMSE}(t)=\sqrt{\frac{\sum_{i=1}^{n}\left(\rho_{i^{\prime}}(t)-\rho_{i}(t)\right)^{2}}{t}}
$$

From (11), root-mean-squared error ' $R M S E$ ' at time ' $t$ ' in the output layer is determined. LLDFFNL algorithmde crease the error by means of changing weights and biases in the network. Subsequently, the weights are updated as,

$$
\Delta \vartheta_{\mu \delta}=-\eta \frac{\partial R M S E(t)}{\partial \vartheta_{\mu \delta}} ; \Delta \vartheta_{\delta_{i}}=-\eta \frac{\partial R M S E(t)}{\partial \vartheta_{\delta_{i}}} ; \Delta \vartheta_{\delta \rho}=-\eta \frac{\partial R M S E(t)}{\partial \vartheta_{\delta \rho}}
$$

From (12), weight values on input ' $\Delta \vartheta_{\mu \delta}$ ', hidden ' $\Delta \vartheta_{\delta_{i}}$ ' and output layer ' $\Delta \vartheta_{\delta \rho}$ ' is updated according to their root-mean-squared error. Here, ' $\eta$ ' refers to the learning rate which controls the change in weight from one iteration to another. It is formulated as,

$$
\gamma_{i}(t) \operatorname{eargminRMSE}(t) \equiv \operatorname{argmin} \sqrt{\frac{\sum_{i=1}^{n}\left(\rho_{i^{\prime}}(t)-\rho_{i}(t)\right)^{2}}{t}}
$$

From (13), the output error ' $\gamma_{i}(t)$ ' of LLDFFNL is minimized to select the best node for routing. The above process of LLDFFNL algorithm is repeated until the root-mean-squared error is lower. The algorithmic process of LLDFFNL is explained in this section. 


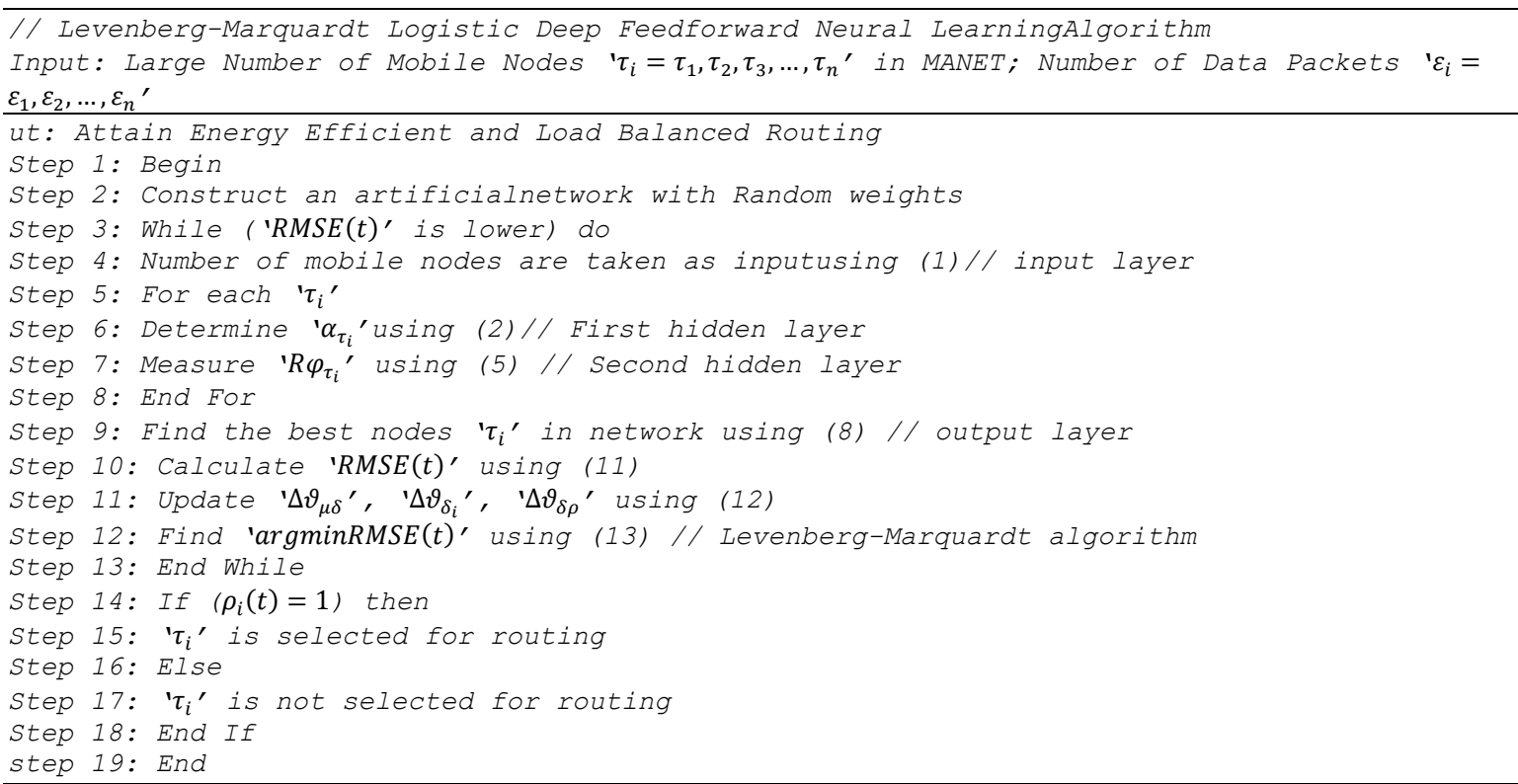

Algorithm 1. Levenberg-Marquardt logistic deep feed forward neural learning

\section{RESULTS AND DISCUSSION}

To estimate the performance of proposed, LLDNL-EELBR Method is implemented in network simulator-3.26 with varying speed of nodes, data rate and number of mobile modes. The performance of LLDNL-EELBR method is calculated in terms of throughput, energy consumption, end to end delay and packet loss rate and the result is compared against with two existing methods namely multipath battery and mobility-aware routing scheme (MBMA-OLSR) and reliable and practical opportunistic routing with gradient forwarding for MANETs named as ORGMA and analyzed with the help of tables and graphs.

\subsection{Simulation environment}

In this simulation, a MANET with 50 to 500 mobile nodes is randomly scattered within a $1200 \mathrm{~m} \mathrm{x}$ $1200 \mathrm{~m}$ area. The nodes are moving in different directions with a speed varying from 0 to $25 \mathrm{~m} / \mathrm{s}$ with the pause time of $10 \mathrm{~s}$. An initial amount of energy is considered as $100 \mathrm{~J}$. The mobile nodes move using random waypoint mobility model. The routing protocol used is DSR and the data packet size is 512 bytes. The mobile nodes are in the transmission range 20 to 200 meters and the MAC layer protocol used is 802.11 . It uses constant bit rate (CBR) traffic and routing bandwidth of $2 \mathrm{Mbps}$. Each simulation has been run 10 times to obtain the optimal results for comparisons. The simulation parameter settings are described in Table 1.

Table 1. Simulation parameters

\begin{tabular}{ll}
\hline \multicolumn{1}{c}{ Simulation Parameters } & \multicolumn{1}{c}{ Values } \\
\hline Simulator & NS 3.26 \\
Number of mobile nodes & $50-500$ \\
Simulation area & $1200 \mathrm{~m} \times 1200 \mathrm{~m}$ \\
Simulation Time & $200 \mathrm{sec}$ \\
Simulation iteration & 10 \\
Initial energy & $100 \mathrm{~J}$ \\
Packet size & $512 \mathrm{bytes}$ \\
Number of data packets & $20,40,60,80,100,120,140,160,180,200$ \\
Transmission range of nodes & $20 \mathrm{~m}$ to $200 \mathrm{~m}$ \\
Movement & Random waypoint \\
Maximum speed & $25 \mathrm{~m} / \mathrm{s}$ \\
Routing protocol & Dynamic source routing (DSR) \\
\hline
\end{tabular}

\subsection{Simulation results}

In this section, the simulation result of LLDNL-EELBR method is compared against MBMA-OLSR and ORGMA. The reported results are obtained with several runs. 


\subsubsection{Effect of throughput}

The throughput is defined as the number of data packets that are successfully reached to the destination at a specific amount of time. The throughput is mathematically obtained using,

$$
\text { Throughput }=\frac{n \varepsilon_{S D}}{t}
$$

From (14), ' $n \varepsilon_{S D}$ ' represents the number of data packets successfully transmitted to the destination without any information loss at time ' $t$ '. The throughput is measured in terms of packets per second (pps). Figure 4 presents the throughput with respect to different number of data packets. The LLDNL-EELBR method achieves 176 pps throughput when taking 200 packets as input whereas MBMA-OLSR, ORGMA gets $166 \mathrm{pps}$ and $170 \mathrm{pps}$. From the average value, the proposed method increases the throughput rate by 16 $\%$ as compared to MBMA-OLSR and $8 \%$ as compared to ORGMA. Hence, LLDNL-EELBR method significantly improves the successful delivery of packets when compared to existing methods.

\subsubsection{Effect of end to end delay}

End to end delay measures the amount of time taken to successfully deliver packets to a destination. Therefore, end to end delay is determined as the distinction between the arrival time and sending time of a packet. It is calculated in terms of milliseconds (ms). The end-to-end delay is calculated as,

$$
\text { EndToendDelay }=a t_{\varepsilon}-s t_{\varepsilon}
$$

From (15), ' $a t_{\varepsilon}$ ' point outs arrival time of a packet whereas ' $s t_{\varepsilon}$ ' represents the sending time of a packet. Figure 5 depicts the comparison of end-to-end delay. The LLDNL-EELBR method obtains $57 \mathrm{~ms}$ end to end delay when considering 100 packets as input whereas MBMA-OLSR, ORGMA attains 64 ms and 62 ms. Clearly, LLDNL-EELBR takes a minimal amount of time to reach destination. As a result, LLDNLEELBR method substantially reduces the end-to-end delay by $17 \%$ when compared to MBMA-OLSR and $13 \%$ as compared to ORGMA.

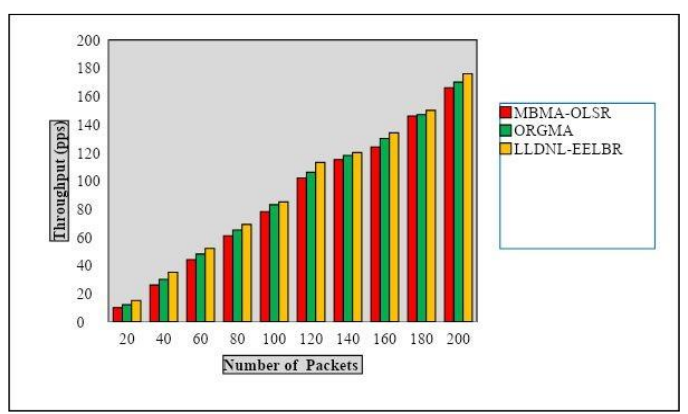

Figure 4. Throughput versus number of packet

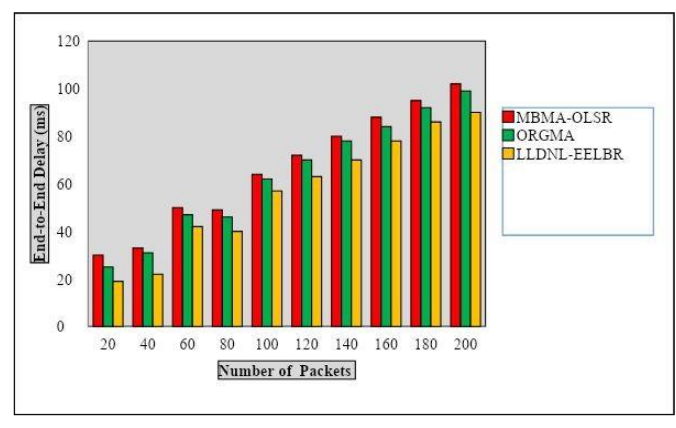

Figure 5. End to end delay versus number of packet

\subsubsection{Effect of packet loss rate}

Packet loss rate measures the ratio between the number of packets lost and the total number of data packets sent. The packet loss rate is determined in percentage (\%). The packet loss rate is estimated as,

$$
\text { PacketLossRate }=\frac{n \varepsilon_{l}}{n \varepsilon_{s}} * 100
$$

From (16), ' $n \varepsilon_{l}$ ' designate the number of dropped and ' $n \varepsilon_{s}$ signifies total number of data packets sent. Figure 6 portrays the impacts of packet loss rate with varying numbers of packets. Obviously, LLDNLEELBR Method gets $14 \%$ packet loss rate whereas MBMA-OLSR, ORGMA acquires 23\% and 18\% respectively. As compared to MBMA-OLSR and ORGMA, the percentage of reduction in packet loss rate of LLDNL-EELBR method is $36 \%$ and $25 \%$ on average. Therefore, the proposed method achieves good reduction in packet rate due to energy limitation and traffic.

\subsubsection{Effect of energy utilization}

The energy utilization determines the amount of energy required to successfully deliver data packets to the destination from a source node. The energy utilization is measured in terms of Joules ( $\mathrm{J}$ ). The energy consumption is calculated as, 


$$
\text { Energy Utilization }=n * \alpha U(S)
$$

From (17), ' $n$ ' is the total number of mobile nodes and ' $\alpha U(S)$ ' represents the energy used by a single node. Figure 7 illustrates energy utilization with respect to various numbers of mobile nodes. LLDNLEELBR Method consumes 48J energy when conducting simulation process with 300 mobile nodes whereas MBMA-OLSR and ORGMA uses $77 \mathrm{~J}$ and $70 \mathrm{~J}$ respectively. Totally, the proposed method minimizes the energy utilization by $33 \%$ compared to MBMA-OLSR and $27 \%$ compared to ORGMA on average. Thus, LLDNL-EELBR considerably reduces the extra amount of energy utilized to rebroadcasting data due to link failure, congestion on mobile nodes and enhances the successful delivery of packets to the destination node. Table 2 clearly summarizes that, LLDNL-EELBR method performs better than other two methods in terms of throughput, delay, packet loss rate and energy consumption.

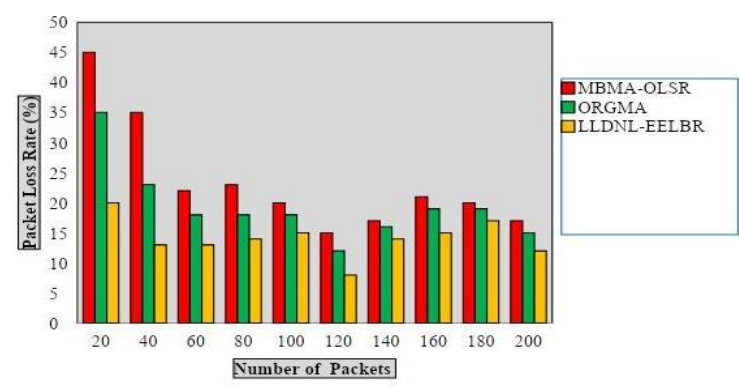

Figure 6. Packet loss rate versus number of packets

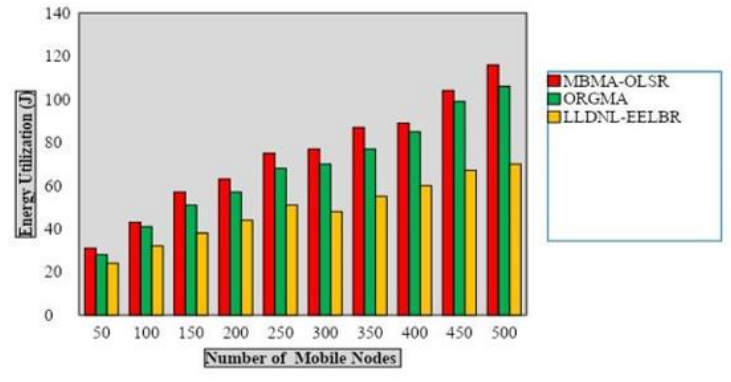

Figure 7. Energy utilization versus number of mobile nodes

Table 2. Result Comparison

\begin{tabular}{lccc}
\hline \multicolumn{1}{c}{ Parameter/Methods } & MBMA-OLSR & ORGMA & LLDNL-EELBR \\
\hline Throughput (pps) & $88(\mathrm{pps})$ & $91(\mathrm{pps})$ & $95(\mathrm{pps})$ \\
End-to-End delay $(\mathrm{ms})$ & $66(\mathrm{~ms})$ & $64(\mathrm{~ms})$ & $57(\mathrm{~ms})$ \\
Packet loss rate $(\%)$ & $24 \%$ & $20 \%$ & $14 \%$ \\
Energy consumption $(\mathrm{J})$ & $74 \mathrm{~J}$ & $68 \mathrm{~J}$ & $49 \mathrm{~J}$ \\
\hline
\end{tabular}

\section{CONCLUSION}

The goal of the proposed LLDNL-EELBR method is enhancing the routing efficiency of MANET through load balancing and energy minimization. By using this method, the successful delivery of data packet increases significantly with a minimal delay by selecting the nodes with a higher residual energy and load. Also, it decreases path failures and traffic congestion because of energy constrictions which automatically reduces the packet loss rate. This method further enhances the stability and reliability of routing by reducing energy consumption thereby increasing the network lifetime. Subsequently, the performance of the proposed method is evaluated in terms of Qos parameters and compared with MBMAOLSR and ORGMA. Simulation result demonstrates that LLDNL-EELBR method gives better performance with an enhancement of throughput and minimizes energy consumption. Future work can be preceded to solve the problem of route path discovery without any link breaks during multipath transmission under different load conditions. In addition, the security is considered in future work for effective transfer of data without prior knowledge of the network or the degree of trustworthiness of the intermediate nodes.

\section{REFERENCES}

[1] W. A. Jabbar, M. Ismail, and R. Nordin, "Energy and mobility conscious multipath routing scheme for route stability and load balancing in MANETs," Simulation Modelling Practice and Theory, vol. 77, pp. 245-271, 2017, doi: 10.1016/j.simpat.2017.07.001.

[2] D. Kang, Hyung-Sin Kim, C. Joo, and S. Bahk," ORGMA: Reliable Opportunistic Routing with Gradient Forwarding for MANETs," Computer Networks, Elsevier, vol. 131, pp. 52-64, 2018, doi: 10.1016/j.comnet.2017.12.001.

[3] H. Amraoui, A. Habbani, and A. Hajami," A Forwarding Game Approach for Reducing Topology Control Traffic in MANETs," Arabian Journal for Science and Engineering, Springer, vol. 43, pp. 6945-6961, 2018, doi: 10.1007/s13369-017-2910-7. 
[4] Y. Chen, E. H. Wu, C. Lin, and G. Chen, "Bandwidth-Satisfied and Coding-Aware Multicast Protocol in MANETs," IEEE Transactions on Mobile Computing, vol. 17, no. 8, pp. 1778-1790, 1 Aug. 2018, doi: 10.1109/TMC.2017.2778262.

[5] T. Singh, J. Singh, and S. Sharma, "Energy efficient secured routing protocol for MANETs," Wireless Network, vol. 23, pp. 1001-1009, 2017, doi: 10.1007/s11276-015-1176-9.

[6] W. A. Jabbar, W. K. Saad, and M. Ismail, "MEQSA-OLSRv2: A Multicriteria-Based Hybrid Multipath Protocol for Energy-Efficient and QoS-Aware Data Routing in MANET-WSN Convergence Scenarios of IoT," IEEE Access, vol. 6, pp. 76546-76572, 2018, doi: 10.1109/ACCESS.2018.2882853.

[7] S. Jamali, L. Rezaei, and S. J. Gudakahriz, "An Energy-efficient Routing Protocol for MANETs: a Particle Swarm Optimization Approach," Journal of Applied Research and Technology, Elsevier, vol. 11, pp. 803-812, 2013.

[8] A. Bhattacharya and K. Sinha, "An efficient protocol for load-balanced multipath routing in mobile ad hoc networks," Ad Hoc Networks, Elsevier, vol. 63, pp. 104-114, Aug. 2017, doi: 10.1016/j.adhoc.2017.05.008.

[9] H. A. Ali, M. F. Areed, and D. I. Elewely, "An on-demand power and load-aware multi-path node-disjoint source routing scheme implementation using NS-2 for mobile ad-hoc networks," Simulation Modelling Practice and Theory, vol. 80, pp. 50-65, Jan. 2018, doi: 10.1016/j.simpat.2017.09.005.

[10] G. Pathak and K. Kumar," Traffic aware load balancing in AOMDV for mobile Ad-hoc networks," Journal of Communications and Information Networks, Springer, vol. 2, pp. 123-130, 2017, doi: 10.1007/s41650-017-0012-z.

[11] J. Zhou, H. Tan, Y. Deng, L. Cui, and D. D. Liu, "Ant colony-based energy control routing protocol for mobile ad hoc networks under different node mobility models," EURASIP Journal on Wireless Communications and Networking, Springer, vol. 105, pp. 1-8, 2016, doi: 10.1186/s13638-016-0600-x.

[12] S. Chettibi and S. Chikhi, "Dynamic fuzzy logic and reinforcement learning for adaptive energy efficient routing in mobile ad-hoc networks," Applied Soft Computing, vol. 38, pp. 321-328, Jan. 2016, doi: 10.1016/j.asoc.2015.09.003.

[13] S. Hao, H. Zhang, and M. Song, "A Stable and Energy-Efficient Routing Algorithm Based on Learning Automata Theory for MANET," in Journal of Communications and Information Networks, vol. 3, no. 2, pp. 43-57, Jun. 2018, doi: 10.1007/s41650-018-0012-7.

[14] M. Rath, B. Pati, B. K. Pattanayak, C. R. Panigrahi, and J. L. Sarkar," Load balanced routing scheme for MANETs with power and delay optimization," International Journal of Communication Networks and Distributed Systems, vol. 19, no. 4, pp. 394-405, 2017, doi: 10.1504/IJCNDS.2017.087386.

[15] M. A. Salem and R. Yadav, "Efficient Load Balancing Routing Technique for Mobile Ad Hoc Networks," International Journal of Advanced Computer Science and Applications, vol. 7, no. 5, pp. 249-254, 2016.

[16] S. Russia and R. Anita, "Joint cost and secured node disjoint energy efficient multipath routing in mobile ad hoc network," Wireless Networks, vol. 23, no. 7, pp. 2307-2316, 2016, doi: 10.1007/s11276-016-1288-x.

[17] R. Havinal, G. V. Attimarad, and M. N. G. Prasad, "MECOR: Minimal Energy Consumption with Optimized Routing in MANET," Wireless personal communication, vol. 88, no. 4, pp. 963-983, 2016, doi: 0.1007/s11277016-3223-y.

[18] S. K. Das and S. Tripathi, "Intelligent energy-aware efficient routing for MANET," Wireless Networks, Springer, vol. 24, no. 4, pp. 1139-1159, 2018, doi: 10.1007/s11276-016-1388-7.

[19] B. H. AlQarni and A. S. AlMogren, "Reliable and Energy Efficient Protocol for MANET Multicasting," Journal of Computer Networks and Communications, 2016, doi: 10.1155/2016/9146168.

[20] A. Taha, R. Alsaqour, M. Uddin, M. Abdelhaq, and T. Saba, "Energy Efficient Multipath Routing Protocol for Mobile Ad-Hoc Network Using the Fitness Function," IEEE Access, vol. 5, pp. 10369-10381, 2017, doi: 10.1109/ACCESS.2017.2707537.

[21] M. M. Afsar and M. Younis, "CRoss-layer design for WSNs with Energy Scavenging and Transfer capabilities (CREST)," Journal of network and computer applications, vol. 145, p. 102390, 2019, doi: 10.1016/j.jnca.2019.06.010.

[22] S. Maurya, V. K. Jain, and D. R. Chowdhury, "Delay aware energy efficient reliable routing for data transmission in heterogeneous mobile sink wireless sensor network," Journal of network and computer applications, vol. 144, pp.118-137, Oct. 2019, doi: 10.1016/j.jnca.2019.06.012.

[23] P. Vijayakumar, R. Rajashree, and P. Sandhya, "A Secure Route Discovery Protocol for AODV Based Mobile Adhoc Networks Using Hyperelliptic Curve Cryptography," Emerging Trends in Electrical, Communications and Information Technologies, vol. 394, pp. 209-217, 2017, doi: 10.1007/978-981-10-1540-3_22.

[24] N. Khatoon, "Mobility Aware Energy Efficient Clustering for MANET: A Bio-Inspired Approach with Particle Swarm Optimization," Wireless Communications and Mobile Computing, vol. 2017, 2017, doi: 10.1155/2017/1903190.

[25] R. N. Jadoon, W. Y. Zhou, I. A. Khan, M. A. Khan, and W. Jadoon, "EEHRT: Energy Efficient Technique for Handling Redundant Traffic in Zone-Based Routing for Wireless Sensor Network," Wireless Communications and Mobile Computing, vol. 2019, 2019, doi: 10.1155/2019/7502140. 